\title{
SGR 0418+5729, Swift J1822.3-1606, and 1E 2259+586 as massive, fast-rotating, highly magnetized white dwarfs
}

\author{
K. Boshkayev, L. Izzo, J. A. Rueda Hernandez, and R. Ruffini
}

\author{
Dipartimento di Fisica and ICRA, Sapienza Università di Roma, P.le Aldo Moro 5, Rome 00185, Italy ICRANet, \\ P.zza della Repubblica 10, Pescara 65122, Italy \\ e-mail: jorge.rueda@icra.it
}

Received 29 November 2012 / Accepted 29 May 2013

\begin{abstract}
Aims. We describe the so-called low magnetic field magnetars, SGR 0418+5729, Swift J1822.3-1606, and the AXP prototype 1E 2259+586 as massive, fast-rotating, highly magnetized white dwarfs.

Methods. We give bounds for the mass, radius, moment of inertia, and magnetic field for these sources by requesting the stability of realistic, general relativistic, uniformly rotating white dwarfs. We also present the theoretical expectation of the infrared, optical, and ultraviolet emission of these objects and show their consistency with the current available observational data.

Results. We improve the theoretical prediction of the lower limit of the spindown rate of SGR 0418+5729; for a white dwarf close to its maximum stable mass we obtain the very stringent interval for the spindown rate of $4.1 \times 10^{-16}<\dot{P}<6 \times 10^{-15}$, where the upper value is the known observational limit. A lower limit has been also set for Swift J1822.3-1606, whose spindown rate is not yet fully confirmed. Our model provides for the source $\dot{P} \geq 2.13 \times 10^{-15}$ if the star is close to its maximum stable mass. We give in addition the frequencies at which absorption features could be present in the spectrum of these sources as the result of the scattering of photons with the quantized electrons by the surface magnetic field.
\end{abstract}

Key words. stars: magnetic field - stars: rotation - white dwarfs - stars: magnetars

\section{Introduction}

Soft gamma-ray repeaters (SGRs) and anomalous X-ray pulsars (AXPs) are a class of compact objects that show interesting observational properties (see, e.g., Mereghetti 2008): rotational periods in the range $P \sim(2-12)$ s, spindown rates $\dot{P} \sim$ $\left(10^{-13}-10^{-10}\right)$, strong outburst of energies $\sim\left(10^{41}-10^{43}\right) \mathrm{erg}$, and in the case of SGRs, giant flares of even large energies $\sim\left(10^{44}-10^{47}\right)$ erg.

The most popular model for the description of SGRs and AXPs, the magnetar model, is based on a neutron star of fiducial parameters $M=1.4 M_{\odot}, R=10 \mathrm{~km}$, and a moment of inertia $I=10^{45} \mathrm{~g} \mathrm{~cm}^{2}$. It needs a neutron star magnetic field larger than the critical field for vacuum polarization $B_{\mathrm{c}}=m_{\mathrm{e}}^{2} c^{3} /(e \hbar)=$ $4.4 \times 10^{13} \mathrm{G}$ in order to explain the observed X-ray luminosity in terms of the release of magnetic energy (Duncan \& Thompson 1992; Thompson \& Duncan 1995). There exist in the literature other models still based on neutron stars but of ordinary fields $B \sim 10^{12} \mathrm{G}$; these models involve either the generation of drift waves in the magnetosphere or the accretion of fallback material via a circumstellar disk (see Malov 2010; Trümper et al. 2013, respectively, and references therein).

Turning to the experimental point of view, the observation of SGR $0418+5729$ with a rotational period of $P=9.08 \mathrm{~s}$, an upper limit of the first time derivative of the rotational period $\dot{P}<6.0 \times 10^{-15}$ (Rea et al. 2010), and an X-ray luminosity of $L_{X}=6.2 \times 10^{31} \mathrm{erg} \mathrm{s}^{-1}$ can be considered as the Rosetta Stone for alternative models of SGRs and AXPs. The inferred upper limit of the surface magnetic field of SGR 0418+5729, $B<7.5 \times 10^{12} \mathrm{G}$; which describes it as a neutron star (see Rea et al. 2010, for details), is well below the critical field. This has challenged the power mechanism based on magnetic field decay in the magnetar scenario.

Alternatively, it has been recently pointed out how the pioneering works of Morini et al. (1988) and Paczynski (1990) on the description of 1E $2259+586$ as a white dwarf can be indeed extended to all SGRs and AXPs. Such white dwarfs were assumed to have fiducial parameters $M=1.4 M_{\odot}, R=10^{3} \mathrm{~km}$, $I=10^{49} \mathrm{~g} \mathrm{~cm}^{2}$, and magnetic fields $B \gtrsim 10^{7} \mathrm{G}$ inferred from the observed rotation periods and spindown rates (Malheiro et al. 2012).

It is remarkable that white dwarfs with large magnetic fields from $10^{7} \mathrm{G}$ all the way up to $10^{9} \mathrm{G}$ have been indeed observed (see e.g., Külebi et al. 2009, 2010a; Kepler et al. 2010, 2013). Also, most of the observed magnetized white dwarfs are massive; e.g., REJ 0317-853 with $M \sim 1.35 M_{\odot}$ and $B \sim$ (1.7-6.6) $\times 10^{8} \mathrm{G}$ (Barstow et al. 1995; Külebi et al. 2010b); PG $1658+441$ with $M \sim 1.31 M_{\odot}$ and $B \sim 2.3 \times 10^{6} \mathrm{G}$ (Liebert et al. 1983; Schmidt et al. 1992); and PG 1031+234 with the highest magnetic field $\sim 10^{9} \mathrm{G}$ (Schmidt et al. 1986; Külebi et al. 2009).

The energetics of SGRs and AXPs, including their steady emission, glitches, and subsequent outburst activities, have been shown to be powered by the rotational energy of the white dwarf (Malheiro et al. 2012). The occurrence of a glitch, the associated sudden shortening of the period, and the corresponding gain of rotational energy can be explained by the release of gravitational energy associated with a sudden contraction and decrease of the moment of inertia of the uniformly rotating white dwarf, which is consistent with the conservation of their angular momentum.

Describing SGR $0418+5729$ as a white dwarf, Malheiro et al. (2012) calculated an upper limit for the magnetic field 
$B<7.5 \times 10^{8} \mathrm{G}$ and show that the X-ray luminosity observed from SGR $0418+5729$ can be well explained as originating from the loss of rotational energy of the white dwarf, leading to a theoretical prediction for the spindown rate

$\frac{L_{X} P^{3}}{4 \pi^{2} I}=1.18 \times 10^{-16} \leq \dot{P}_{\text {SGR } 0418+5729}<6.0 \times 10^{-15}$,

where the lower limit was established by assuming that the observed X-ray luminosity of SGR $0418+5729$ coincides with the rotational energy loss of the white dwarf. As we show below, these predictions can be improved by considering realistic white dwarf parameters instead of fiducial values. It is important to mention at this point that, after the submission of this work, Rea et al. (2013) presented the X-ray timing analysis of the long-term monitoring of SGR 0418+5729 with RXTE, Swift, Chandra, and $X M M-N e w t o n$, which allowed the determination of the spindown rate of SGR $0418+5729, \dot{P}=4 \times 10^{-15}$. These results confirm both our prediction given by Eq. (1) and the more stringent limits presented in this work in Sect. 4.4 and given by Eq. (10), which are to be considered to be predictions of the white dwarf model because they were presented in advance of the observational results given in (Rea et al. 2013).

The situation has become even more striking considering the X-ray timing monitoring with Swift, RXTE, Suzaku, and XMM-Newton satellites of the recently discovered SGR Swift J1822.3-1606 (Rea et al. 2012). The rotation period $P=8.437 \mathrm{~s}$ and the spindown rate $\dot{P}=9.1 \times 10^{-14}$ were obtained. Assuming a neutron star of fiducial parameters, a magnetic field $B=2.8 \times 10^{13} \mathrm{G}$ is inferred, which is again in contradiction to a magnetar explanation for this source.

We recently computed (Boshkayev et al. 2013) general relativistic, uniformly rotating white dwarfs within the Hartle's formalism (Hartle 1967). We used the relativistic FeynmanMetropolis-Teller equation of state (Rotondo et al. 2011), which generalizes the traditionally used equation of state of Salpeter (1961). It was shown there that rotating white dwarfs can be stable up to rotation periods close to $0.3 \mathrm{~s}$ (see Boshkayev et al. 2013 and Sect. 3 for details). This range of stable rotation periods for white dwarfs amply covers the observed rotation rates of SGRs and AXPs, $P \sim(2-12) \mathrm{s}$.

The aim of this work is to give a detailed description of the so-called low magnetic field magnetars, SGR 0418+5729 and Swift J1822.3-1606, as massive, fast-rotating, highly magnetized white dwarfs. In addition to these two sources, we also present a similar analysis of the AXP prototype 1E $2259+586$, which is the source on which Morini et al. (1988) and Paczynski (1990) proposed the idea of a description of AXPs based on white dwarfs. We thus extend the work of Malheiro et al. (2012) by using precise white dwarf parameters recently obtained by Boshkayev et al. (2013) for general relativistic, uniformly rotating white dwarfs. We present an analysis of the expected optical and near-infrared (near-IR) emission from these sources within the white dwarf model and confront the results with the observational data.

\section{Rotation-powered white dwarfs}

The loss of rotational energy associated with the spindown of the white dwarf is given by

$\left|\dot{E}_{\text {rot }}\right|=4 \pi^{2} I \frac{\dot{P}}{P^{3}}=3.95 \times 10^{50} I_{49} \frac{\dot{P}}{P^{3}} \quad \mathrm{erg}^{-1}$,

where $I_{49}$ is the moment of inertia of the white dwarf in units of $10^{49} \mathrm{~g} \mathrm{~cm}^{2}$. This rotational energy loss amply justifies the steady X-ray emission of all SGRs and AXPs (Malheiro et al. 2012).

The upper limit on the magnetic field obtained by requesting that the rotational energy loss due to the dipole field be smaller than the electromagnetic emission of the magnetic dipole is given by (see, e.g. Ferrari \& Ruffini 1969)

$B=\sqrt{\frac{3 c^{3}}{8 \pi^{2}} \frac{I}{\bar{R}^{6}} P \dot{P}}=3.2 \times 10^{15} \sqrt{\frac{I_{49}}{\bar{R}_{8}^{6}} P \dot{P}} \quad \mathrm{G}$,

where $\bar{R}_{8}$ is the mean radius of the white dwarf in units of $10^{8} \mathrm{~cm}$. The mean radius is given by $\bar{R}=\left(2 R_{\mathrm{eq}}+R_{\mathrm{p}}\right) / 3$ (see, e.g., Hartle $\&$ Thorne 1968$)$ with $R_{\text {eq }}$ and $R_{\mathrm{p}}$ the equatorial and polar radius of the star.

It is clear that the specific values of the rotational energy loss and the magnetic field depend on observed parameters, such as $P$ and $\dot{P}$, and on model parameters, such as the mass, moment of inertia, and mean radius of the rotating white dwarf. Equation (3) gives information only on the dipole component of the magnetic field, but there is the possibility that close to the star surface contributions from higher multipoles could also be important. As shown by Qadir et al. (1980), the presence of higher electromagnetic multipoles increases the pulsar braking index to values larger than the traditional value $n=3$ of the magneto-dipole radiation.

\section{Structure and stability of rotating white dwarfs}

The rotational stability of fast-rotating white dwarfs was implicitly assumed by Malheiro et al. (2012). The crucial question of whether rotating white dwarfs can or cannot attain rotation periods as short as the ones observed in SGRs and AXPs has been recently addressed by Boshkayev et al. (2013). The properties of uniformly rotating white dwarfs were computed within the framework of general relativity through the Hartle's formalism (Hartle 1967). The equation of state for cold white dwarf matter is based on the relativistic Feynman-Metropolis-Teller treatment (Rotondo et al. 2011), which generalizes the equation of state of Salpeter (1961). The stability of rotating white dwarfs was analyzed taking into account the mass-shedding limit, inverse $\beta$-decay, and pycnonuclear instabilities, as well as the secular axisymmetric instability, with the latter determined by the turning point method of Friedman et al. (1988) (see Fig. 1 and Boshkayev et al. 2013, for details).

The minimum rotation period $P_{\min }$ of white dwarfs is obtained for a configuration rotating at Keplerian angular velocity at the critical inverse $\beta$-decay density; this is the configuration lying at the crossing point between the mass-shedding and inverse $\beta$-decay boundaries. The numerical values of the minimum rotation period $P_{\min } \approx(0.3,0.5,0.7,2.2)$ s were found for helium, carbon, oxygen, and iron white dwarfs, respectively (Boshkayev et al. 2013). As a byproduct, these values show that indeed all SGRs and AXPs can be described as rotating white dwarfs because their rotation periods are in the range $2 \lesssim P \lesssim 12 \mathrm{~s}$.

The relatively long minimum period of rotating ${ }^{56} \mathrm{Fe}$ white dwarfs, $P_{\min } \approx 2.2 \mathrm{~s}$, which lies just at the lower edge of the observed range of rotation periods of SGRs and AXPs, reveals crucial information on the chemical composition of SGRs and AXPs, namely, that they are very likely made of elements lighter than iron, such as carbon or oxygen.

It can be seen from Fig. 1 that every $\Omega=2 \pi / P$ constant sequence intersects the stability region of general relativistic, uniformly rotating white dwarfs $\left(M-R_{\text {eq }}\right.$ curves inside the shaded 


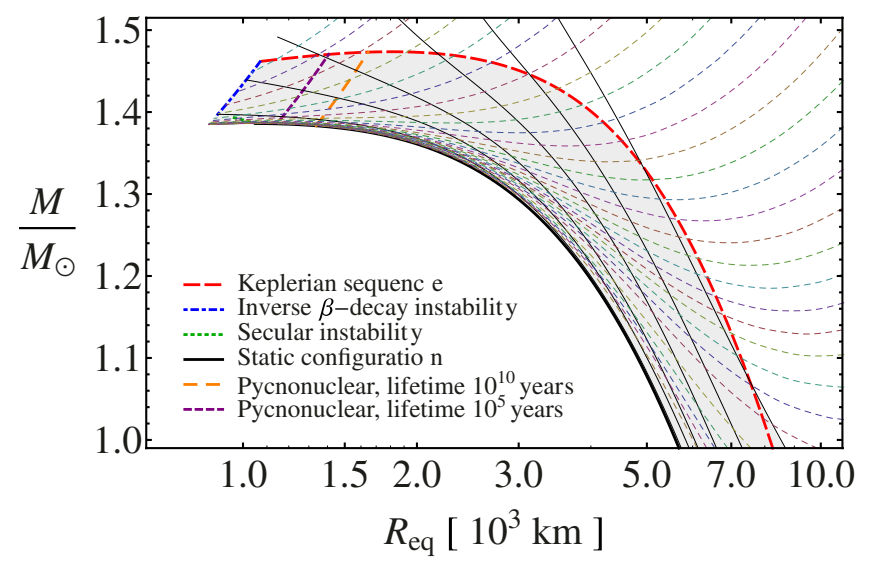

Fig. 1. Mass versus equatorial radius of rotating ${ }^{12} \mathrm{C}$ white dwarfs (Boshkayev et al. 2013). The solid black curves correspond to $J=$ constant sequences, where the static case $J=0$ is the thickest one. The colored thin dashed curves correspond to $P=$ constant sequences. The Keplerian sequence is the red thick dashed curve, the blue thick dotteddashed curve is the inverse $\beta$ instability boundary, and the green thick dotted curve is the axisymmetric instability line. The orange and purple dashed boundaries correspond to the pycnonuclear $\mathrm{C}+\mathrm{C}$ fusion densities with reaction mean times $\tau_{\text {pyc }}=10 \mathrm{Gyr}$ and $0.1 \mathrm{Myr}$, respectively. The gray-shaded region is the stability region of rotating white dwarfs.

region of Fig. 1) in two points. These two points determine the minimum(maximum) mass $M_{\min , \max }$ and maximum(minimum) equatorial radius $R_{\mathrm{eq}}^{\max , \min }$ for the stability of a white dwarf rotating at the given angular velocity. Associated with the boundary values $M_{\min , \max }$ and $R_{\mathrm{eq}}^{\max \text { min }}$, we can obtain the corresponding bounds for the moment of inertia of the white dwarf, $I_{\max , \min }$, respectively.

We turn now to a specific analysis of the two sources, SGR 0418+5729 and SGR 1822-1606.

\section{SGR $0418+5729$}

\subsection{Bounds on the white dwarf parameters}

SGR $0418+5729$ has a rotational period of $P=9.08 \mathrm{~s}$, and the upper limit of the spindown rate $\dot{P}<6.0 \times 10^{-15}$ was obtained by Rea et al. (2010). The corresponding rotation angular velocity of the source is $\Omega=2 \pi / P=0.69 \mathrm{rads}^{-1}$. We show in Table 1 bounds for the mass, equatorial radius, mean radius, and moment of inertia of SGR $0418+5729$, which were obtained by the request of the rotational stability of the rotating white dwarf for selected chemical compositions, as described in Sect. 3. Hereafter we consider only general relativistic, rotating carbon white dwarfs.

\subsection{Solidification and glitches}

It has been shown by Malheiro et al. (2012) that the massive white dwarfs consistent with SGRs and AXPs possibly behave as solids since the internal temperature of the white dwarf $\left(\sim 10^{7} \mathrm{~K}\right)$ is very likely lower than the crystallization temperature (see, e.g., Shapiro \& Teukolsky 1983; Usov 1994)

$T_{\text {cry }} \simeq 2.3 \times 10^{5} \frac{Z^{2}}{A^{1 / 3}}\left(\frac{\bar{\rho}}{10^{6} \mathrm{~g} \mathrm{~cm}^{-3}}\right)^{1 / 3} \mathrm{~K}$,

where $(Z, A)$ and $\bar{\rho}$ denote the chemical composition and mean density, respectively.
This fact introduces the possibility in the white dwarf to observe sudden changes in the period of rotation, namely, glitches. The expected theoretical values of the fractional change of periods of massive white dwarfs have been shown to be consistent with the values observed in many SGRs and AXPs (Malheiro et al. 2012).

From the bounds of $M$ and $R_{\text {eq }}$ we obtain that the mean density of SGR $0418+5729$ must be in the range $2.3 \times 10^{6} \lesssim \bar{\rho} \lesssim$ $5.7 \times 10^{8} \mathrm{~g} \mathrm{~cm}^{3}$. Correspondingly, the crystallization temperature is comprised in the range $4.8 \times 10^{6} \mathrm{~K} \lesssim T_{\text {cry }} \lesssim 3.0 \times 10^{7} \mathrm{~K}$, where the lower and upper limits correspond to the configurations of minimum and maximum mass, respectively.

The crystallization temperature obtained here indicates that SGR $0418+5729$ should behave as a rigid solid body and therefore glitches during the rotational energy loss, accompanied by radiative events, could happen. Starquakes leading to glitches in the white dwarf will occur with a recurrence time (see, e.g., Baym \& Pines 1971; Usov 1994; Malheiro et al. 2012)

$\delta t_{\mathrm{q}}=\frac{2 \mathcal{D}^{2}}{\mathcal{B}} \frac{|\Delta P| / P}{\left|\dot{E}_{\text {rot }}\right|}$,

where

$\mathcal{B}=0.33 \frac{4 \pi}{3} R_{\mathrm{c}}^{3} e^{2} Z^{2}\left(\frac{\bar{\rho}_{\mathrm{c}}}{A m_{p}}\right)^{4 / 3}, \quad \mathcal{D}=\frac{3}{25} \frac{G M_{\mathrm{c}}^{2}}{R_{\mathrm{c}}}$,

with $\dot{E}_{\text {rot }}$ the loss of rotational energy given by Eq. (2), $M_{\mathrm{c}}, R_{\mathrm{c}}$, and $\bar{\rho}_{\mathrm{c}}$ are the mass, radius, and mean density of the solid core, and $m_{\mathrm{p}}$ is the proton mass.

For the minimum and maximum mass configurations and the upper limit of the spindown rate $\dot{P}<6 \times 10^{-15}$, we obtain a lower limit for recurrence time of starquakes:

$\delta t_{\mathrm{q}}>\left\{\begin{array}{lll}4.2 \times 10^{9}(|\Delta P| / P) & \mathrm{yr}, & M=M_{\min } \\ 2.0 \times 10^{12}(|\Delta P| / P) & \mathrm{yr}, & M=M_{\max }\end{array}\right.$

For typical fractional change of periods $|\Delta P| / P=10^{-6}$, which are observed in SGRs and AXPs, we obtain $\delta t_{\mathrm{q}}>4 \times 10^{3} \mathrm{yr}$ and $\delta t_{\mathrm{q}}>2 \times 10^{6} \mathrm{yr}$, for $M_{\min }$ and $M_{\max }$, respectively. These very long starquake recurrent times are in agreement with the possibility that SGR $0418+5729$ is an old white dwarf whose magnetospheric activity is settling down, in line with its relatively low spindown rate, magnetic field, and high efficiency parameter $L_{\mathrm{X}} / \dot{E}_{\text {rot }}$, with respect to the values of other SGRs and AXPs (see e.g. Fig. 9 in Malheiro et al. 2012).

\subsection{Rotation power and magnetic field}

Introducing the values of $P$ and the upper limit $\dot{P}$ into Eq. (2), we obtain an upper limit for the rotational energy loss

$\left|\dot{E}_{\text {rot }}\right|<\left\{\begin{array}{lll}9.1 \times 10^{32} & \text { erg s}^{-1}, & M=M_{\max } \\ 4.5 \times 10^{34} & \text { erg s}^{-1}, & M=M_{\min }\end{array}\right.$

which for any possible mass is larger than the observed X-ray luminosity of SGR $0418+5729, L_{X}=6.2 \times 10^{31} \mathrm{erg} \mathrm{s}^{-1}$, assuming a distance of $2 \mathrm{kpc}$ (Rea et al. 2010).

The corresponding upper limits on the surface magnetic field of SGR $0418+5729$, which are obtained from Eq. (3), are (see also Table 1)

$B<B_{\min , \max }^{\text {upper }}=\left\{\begin{array}{lll}1.2 \times 10^{7} & \mathrm{G}, & M=M_{\min } \\ 3.5 \times 10^{8} & \mathrm{G}, & M=M_{\max }\end{array}\right.$ 
Table 1. Bounds on the properties of SGR 0418+5729.

\begin{tabular}{lcccccccccc}
\hline \hline Composition & $M_{\min }$ & $M_{\max }$ & $R_{\mathrm{eq}}^{\min }$ & $R_{\mathrm{eq}}^{\max }$ & $\bar{R}_{\min }$ & $\bar{R}_{\max }$ & $I_{48}^{\min }$ & $I_{50}^{\max }$ & $B_{\min }^{\text {upper }}$ & $B_{\max }^{\text {upper }}$ \\
\hline Helium & 1.18 & 1.41 & 1.16 & 6.88 & 1.15 & 6.24 & 3.59 & 1.48 & 1.18 & 2.90 \\
Carbon & 1.15 & 1.39 & 1.05 & 6.82 & 1.05 & 6.18 & 2.86 & 1.42 & 1.19 & 3.49 \\
Oxygen & 1.14 & 1.38 & 1.08 & 6.80 & 1.08 & 6.15 & 3.05 & 1.96 & 1.42 & 3.30 \\
Iron & 0.92 & 1.11 & 2.21 & 6.36 & 2.21 & 5.75 & 12.9 & 1.01 & 1.25 & 0.80 \\
\hline
\end{tabular}

Notes. The masses $M_{\text {min,max }}$ are in $M_{\odot}$, the equatorial $R_{\mathrm{eq}}^{\min \text {,max }}$ and mean $\bar{R}_{\min , \max }$ radii area in units of $10^{8} \mathrm{~cm}$, the moments of inertia $I_{48}^{\min }$ and $I_{50}^{\max }$ are in units of $10^{48} \mathrm{~g} \mathrm{~cm}^{2}$ and $10^{50} \mathrm{~g} \mathrm{~cm}^{2}$, respectively. The magnetic fields $B_{\min }^{\text {upper }}$ and $B_{\max }^{\text {upper }}$ are in units of $10^{7} \mathrm{G}$ and $10^{8} \mathrm{G}$, respectively.

The above maximum possible value of the surface magnetic field of SGR $0418+5729$ obtained for the maximum possible mass of a white dwarf with rotation period $9.08 \mathrm{~s}, B<3.49 \times 10^{8} \mathrm{G}$ is even more stringent and improves the previous value given by Malheiro et al. (2012), $B<7.5 \times 10^{8} \mathrm{G}$, based on fiducial white dwarf parameters.

The presence of the magnetic field quantizes the electron energy spectrum, and thus absorption features generated by the scattering of electrons with photons can be observed at frequencies of the order of

$v_{\mathrm{cyc}, \mathrm{e}}=\frac{e B}{2 \pi m_{\mathrm{e}} c}=\left\{\begin{array}{lll}3.4 \times 10^{13} & \mathrm{~Hz}, & M=M_{\min } \\ 9.8 \times 10^{14} & \mathrm{~Hz}, & M=M_{\max },\end{array}\right.$

corresponding to wavelengths 8.9 and $0.3 \mu \mathrm{m}$, respectively.

\subsection{Prediction of the spindown rate}

Assuming that the observed X-ray luminosity is lower than $\left|\dot{E}_{\text {rot }}\right|$, we obtain the lower limit for the spindown rate,

$\dot{P}>\frac{L_{X} P^{3}}{4 \pi^{2} I}= \begin{cases}8.3 \times 10^{-18}, & M=M_{\min } \\ 4.1 \times 10^{-16}, & M=M_{\max },\end{cases}$

which in the case of the white dwarf with the maximum possible mass is more stringent than the value reported by Malheiro et al. (2012), $\dot{P}=1.18 \times 10^{-16}$, for a massive white dwarf of fiducial parameters.

\subsection{Optical spectrum and luminosity}

Durant et al. (2011) observed SGR 0418+5729 with the two wide filters $F 606 W$ and $F 110 W$ of the Hubble Space Telescope within the positional error circle derived from Chandra observations of the field of SGR $0418+5729$ (Rea et al. 2010). They derive the upper limits of the apparent magnitudes, $m_{F 606 W}>28.6$ and $m_{F 110 W}>27.4$ (Vega system). The approximate distance to the source is $d=2 \pm 0.5 \mathrm{kpc}$ (Durant et al. 2011). Assuming an interstellar extinction obtained from the $N_{H}$ column absorption value observed in the X-ray data, $A_{V}=0.7$, Durant et al. (2011) obtained the corresponding luminosity upper bounds $L_{F 606 W}<$ $5 \times 10^{28} \mathrm{erg} \mathrm{s}^{-1}$ and $L_{F 110 W}<6 \times 10^{28} \mathrm{erg} \mathrm{s}^{-1}$.

We used here a similar method, i.e. computing the interstellar extinction values for the $V$-band from the $N_{H}$ column absorption value observed in the X-ray data, $N_{H}=1.5 \times 10^{21} \mathrm{~cm}^{-2}$ (Rea et al. 2010), and then using the empirical formula described in Predehl \& Schmitt (1995). Then we extrapolated the extinction to the other filters by using the method delineated in Cardelli et al. (1989). Since the F606W and the F110W are well approximated by the $V$ and $J$-band, we obtained for the extinction values $A_{F 606 W}=0.83$ and $A_{F 110 W}=0.235$ respectively. The corresponding luminosity upper bounds are, consequently, $L_{F 606 W}<6.82 \times 10^{28} \mathrm{erg} \mathrm{s}^{-1}$ and $L_{F 110 W}<3.05 \times 10^{28} \mathrm{erg} \mathrm{s}^{-1}$.
An estimate of the effective surface temperature can be obtained by approximating the spectral luminosity in these bands by the blackbody luminosity

$L=4 \pi R^{2} \sigma T^{4}$,

where $\sigma=5.67 \times 10^{-5} \mathrm{erg} \mathrm{cm}^{-2} \mathrm{~s}^{-1} \mathrm{~K}^{-4}$ is the Stefan-Boltzmann constant. For a white dwarf of fiducial radius $R=10^{8} \mathrm{~cm}$, the upper limits for the surface temperature, $T<9.6 \times 10^{3} \mathrm{~K}$ and $T<9.2 \times 10^{3} \mathrm{~K}$, can be obtained for the $F 110 \mathrm{~W}$ and $F 606 \mathrm{~W}$ filters, replacing the upper limits for $L_{F 110 W}$ and $L_{F 606 W}$ in Eq. (11). These bounds of the surface temperature of the white dwarf can be improved by using the explicit dependence on the radius of the blackbody surface temperature for each filter. The blackbody flux at a given frequency $v$ in the source frame is given by

$v f_{v}=\pi \frac{2 h}{c^{2}} \frac{v^{4}}{\exp [h v /(k T)]-1}$,

where $h, k$, and $v$ are the Planck constant, the Boltzmann constant, and the spectral frequency, respectively. From this expression we can obtain the temperature as a function of the frequency, the observed flux, the distance $d$, and radius $R$ of the blackbody source

$T=\frac{h v}{k \ln \left(1+\frac{\pi 2 h v^{4} R^{2}}{c^{2} d^{2} F_{v, \text { obs }}}\right)}$,

where we used the relation between the flux in the observed and source frames, $F_{v, \text { obs }}=(R / d)^{2} v f_{v}$.

The observed fluxes, in units of erg, $\mathrm{cm}^{-2}, \mathrm{~s}^{-1}$, corrected for the extinction are given by

$F_{v, \text { obs }}^{F 606 W}=3.6 \times 10^{-20} v_{F 606 W} \times 10^{-0.4\left(m_{F 606 W}-A_{F 606 W}\right)}$,

and

$F_{v, \mathrm{obs}}^{F 110 W}=1.8 \times 10^{-20} v_{F 110 W} \times 10^{-0.4\left(m_{F 110 W}-A_{F 110 W}\right)}$,

where $v_{F 606 W}=5.1 \times 10^{14} \mathrm{~Hz}$ and $v_{F 110 \mathrm{~W}}=2.6 \times 10^{14} \mathrm{~Hz}$ are the pivot frequencies of the $F 606 \mathrm{~W}$ and $F 110 \mathrm{~W}$ filters, respectively.

Introducing the upper limits of the apparent magnitudes of Durant et al. (2011) with the extinction values computed in this work, Eq. (13) gives the upper bounds on the temperature

$T<\left\{\begin{array}{lll}1.3 \times 10^{4}\left[\ln \left(1+0.44 R_{8}^{2}\right)\right]^{-1} & \mathrm{~K}, & F 110 W \\ 2.4 \times 10^{4}\left[\ln \left(1+6.35 R_{8}^{2}\right)\right]^{-1} & \mathrm{~K}, & F 606 W\end{array}\right.$,

where $R_{8}^{2}$ is the radius of the white dwarf in units of $10^{8} \mathrm{~cm}$ and, following Durant et al. (2011), we approximated the band integrated flux as $v_{\mathrm{c}} F_{v}$, with $v_{\mathrm{c}}$ the pivot wavelength of the corresponding band filter.

In Fig. 2, we show the constraints on the $T-R$ relation obtained from Eq. (16). We used the range of radii defined by the 
K. Boshkayev et al.: SGR 0418+5729, Swift J1822.3-1606, and 1E 2259 as white dwarfs

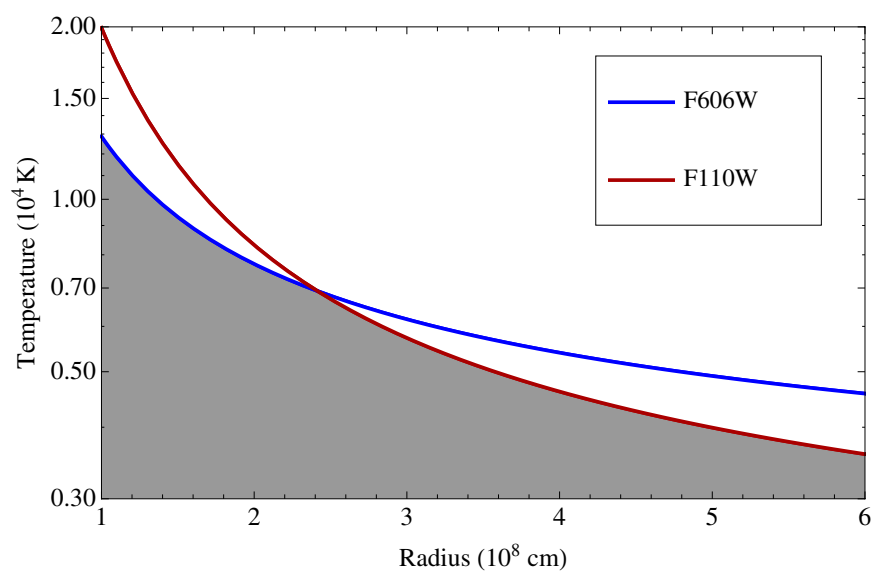

Fig. 2. Temperature-radius constraint given by Eq. (16). The gray region corresponds to the possible values for the temperature and the radius of the white dwarf. The range of radii corresponds to the one defined by the minimum and maximum mean radius of SGR 0418+5729, which was inferred from the white dwarf stability analysis and summarized in Table 1.

minimum and maximum radius of SGR $0418+5729$, which was inferred from the white dwarf stability analysis and summarized in Table 1.

Malheiro et al. (2012) obtained for a white dwarf of fiducial parameters the upper limits for the white dwarf surface temperature, $T<3.14 \times 10^{4}$. We now improve these bounds on the surface temperature using realistic white dwarf parameters. From the minimum and maximum values we obtained for the mean radius of SGR $0418+5729$ (see Table 1) we obtain for the $F 110 W$ filter

$T_{F 110 W}<\left\{\begin{array}{lll}4.3 \times 10^{3} & \mathrm{~K}, & M=M_{\min } \\ 3.2 \times 10^{4} & \mathrm{~K}, & M=M_{\max },\end{array}\right.$

and for the $F 606 W$ filter

$T_{F 606 W}<\left\{\begin{array}{lll}4.4 \times 10^{3} & \mathrm{~K}, & M=M_{\min } \\ 1.2 \times 10^{4} & \mathrm{~K}, & M=M_{\max }\end{array}\right.$

It is clear that these constraints are in agreement with a model based on a massive fast-rotating highly magnetic white dwarf for SGR 0418+5729. It is appropriate to recall in this respect some of the observed temperatures of massive isolated white dwarfs, $1.14 \times 10^{4} \mathrm{~K} \leq T \leq 5.52 \times 10^{4} \mathrm{~K}$, as shown in Table 1 in Ferrario et al. (2005). It is also worth recalling the optical observations of 4U $0142+61$ of Hulleman et al. (2000), where the photometric results of the field of $4 \mathrm{U} 0142+61$ at the 60-inch telescope on Palomar Mountain were found to be in agreement with a $1.3 M_{\odot}$ white dwarf with a surface temperature $\sim 4 \times 10^{5} \mathrm{~K}$.

We show in Fig. 3 the expected optical magnitudes of a white dwarf with surface temperature $T=10^{4} \mathrm{~K}$ and radius $R=1.5 \times$ $10^{8} \mathrm{~cm}$, located at a distance of $2 \mathrm{kpc}$. This radius corresponds to the upper limit that is given by the gray region shown in Fig. 2 for this specific value of the temperature.

\section{Swift J1822.3-1606}

\subsection{Bounds of the white dwarf parameters}

Swift J1822.3-1606 (or SGR 1822-1606) was discovered in July 2011 by the Swift Burst Alert Telescope (BAT). Recent X-ray timing monitoring with Swift, RXTE, Suzaku, and

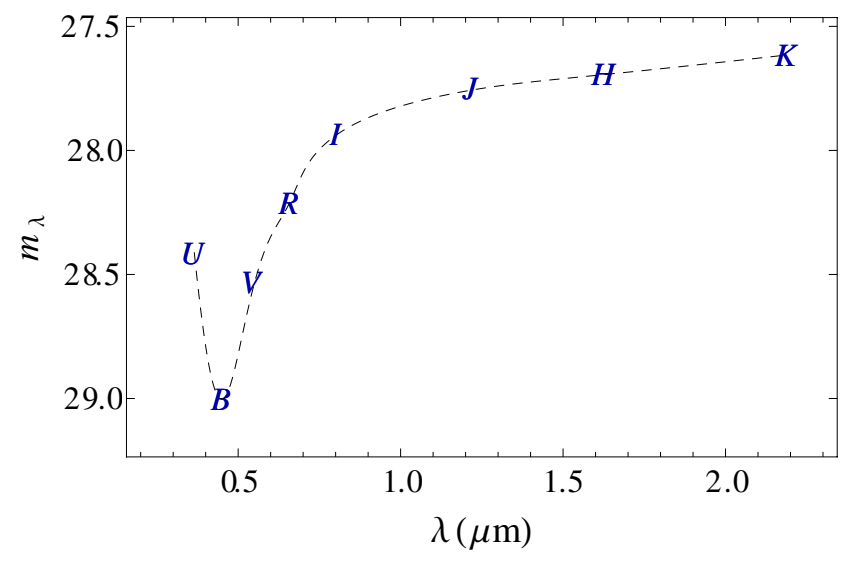

Fig. 3. Expected optical magnitudes of SGR 0418+5729 obtained assuming a simple blackbody for the spectral emission from a white dwarf with surface temperature $T=10^{4} \mathrm{~K}$ and a radius of $1.5 \times 10^{8} \mathrm{~cm}$, according to the constraints shown in Fig. 2.

XMM-Newton satellites found that SGR 1822-1606 rotates with a period of $P=8.44$ s and slows down at a rate $\dot{P}=9.1 \times 10^{-14}$ (see Rea et al. 2012, for details). The corresponding rotation angular velocity of the source is $\Omega=2 \pi / P=0.74 \mathrm{rad} \mathrm{s}^{-1}$. Bounds for the mass, equatorial radius, and moment of inertia of SGR $0418+5729$ obtained by the request of the rotational stability of the rotating white dwarf, as described in Sect. 4, are shown in Table 2.

\subsection{Solidification and glitches}

The mean density of SGR $1822-1606$ is in the range $2.7 \times 10^{6} \lesssim$ $\bar{\rho} \lesssim 5.7 \times 10^{8} \mathrm{~g} \mathrm{~cm}^{3}$. The crystallization temperature for such a range following Eq. (4) is then in the range $5.0 \times 10^{6} \mathrm{~K} \lesssim T_{\text {cry }} \lesssim$ $3.0 \times 10^{7} \mathrm{~K}$, which indicates that SGR $1822-1606$ will likely behave as a rigid solid body.

For the minimum and maximum mass configurations and the spindown rate $\dot{P}=9.1 \times 10^{-14}$, we obtain a lower limit for recurrence time of starquakes,

$\delta t_{\mathrm{q}}>\left\{\begin{array}{lll}2.6 \times 10^{8}(|\Delta P| / P) & \mathrm{yr}, & M=M_{\min } \\ 1.1 \times 10^{11}(|\Delta P| / P) & \mathrm{yr}, & M=M_{\max }\end{array}\right.$

which for a typical fractional change of period $|\Delta P| / P \sim 10^{-6}$ gives $\delta t_{\mathrm{q}}>3 \times 10^{2} \mathrm{yr}$ and $\delta t_{\mathrm{q}}>10^{5} \mathrm{yr}$ for $M_{\min }$ and $M_{\max }$, respectively. The long recurrence time for starquakes obtained in this case confirms the similarities between SGR 1822-1606 and SGR $0418+5729$ as old objects with a settling down magnetospheric activity.

\subsection{Rotation power and magnetic field}

Using the observed values of $P$ and $\dot{P}$, we obtain from Eq. (2) a rotational energy loss

$\left|\dot{E}_{\text {rot }}\right| \approx\left\{\begin{array}{lll}1.7 \times 10^{34} & \mathrm{erg} \mathrm{s}^{-1}, & M=M_{\max } \\ 7.9 \times 10^{35} & \mathrm{erg} \mathrm{s}^{-1}, & M=M_{\min },\end{array}\right.$

which amply justifies the observed X-ray luminosity of SGR 1822-1606, $L_{X}=4 \times 10^{32} \mathrm{erg} \mathrm{s}^{-1}$, which was obtained assuming a distance of $5 \mathrm{kpc}$ (Rea et al. 2012). 
Table 2. Bounds on the properties of Swift J1822.3-1606.

\begin{tabular}{lcccccccccc}
\hline \hline Composition & $M_{\min }$ & $M_{\max }$ & $R_{\mathrm{eq}}^{\min }$ & $R_{\mathrm{eq}}^{\max }$ & $\bar{R}_{\min }$ & $\bar{R}_{\max }$ & $I_{48}^{\min }$ & $I_{50}^{\max }$ & $B_{\min }$ & $B_{\max }$ \\
\hline Helium & 1.21 & 1.41 & 1.16 & 6.61 & 1.15 & 5.99 & 3.59 & 1.38 & 4.84 & 1.09 \\
Carbon & 1.17 & 1.39 & 1.05 & 6.55 & 1.05 & 5.93 & 2.86 & 1.32 & 4.87 & 1.31 \\
Oxygen & 1.16 & 1.38 & 1.08 & 6.53 & 1.08 & 5.91 & 3.05 & 1.83 & 5.80 & 1.24 \\
Iron & 0.95 & 1.11 & 2.21 & 6.11 & 2.20 & 5.53 & 12.9 & 0.94 & 5.09 & 0.30 \\
\hline
\end{tabular}

Notes. The masses $M_{\text {min,max }}$ are in $M_{\odot}$, the equatorial $R_{\mathrm{eq}}^{\min \text {,max }}$ and mean $\bar{R}_{\text {min,max }}$ radii area in units of $10^{8} \mathrm{~cm}$, the moments of inertia $I_{48}^{\min }$ and $I_{50}^{\max }$ are in units of $10^{48} \mathrm{~g} \mathrm{~cm}^{2}$ and $10^{50} \mathrm{~g} \mathrm{~cm}^{2}$, respectively. The magnetic fields $B_{\min }$ and $B_{\max }$ are in units of $10^{7} \mathrm{G}$ and $10^{9} \mathrm{G}$, respectively.

The surface magnetic field of SGR 1822.3-1606, as given by Eq. (3), is then between the values (see Table 2)

$B=\left\{\begin{array}{lll}4.9 \times 10^{7} & \mathrm{G}, & M=M_{\min } \\ 1.3 \times 10^{9} & \mathrm{G}, & M=M_{\max }\end{array}\right.$

Corresponding to the above magnetic fields, the electron cyclotron frequencies are

$v_{\mathrm{cyc}, \mathrm{e}}=\frac{e B}{2 \pi m_{\mathrm{e}} c}=\left\{\begin{array}{lll}1.4 \times 10^{14} & \mathrm{~Hz}, & M=M_{\min } \\ 3.6 \times 10^{15} & \mathrm{~Hz}, & M=M_{\max }\end{array}\right.$

which correspond to wavelengths 2.2 and $0.08 \mu \mathrm{m}$, respectively.

\subsection{Optical spectrum and luminosity}

Rea et al. (2012) observed the field of SGR 1822-1606 with the Gran Telescopio Canarias (GranTeCan) within the Swift-XRT position (Pagani et al. 2011). Three sources ( $S 1, S 2$, and $S 3$ ) were detected with the Sloan $z$ filter with corresponding $z$-band magnitudes $m_{z, S 1}=18.13 \pm 0.16, m_{z, S 2}=20.05 \pm 0.04$, and $m_{z, S 3}=19.94 \pm 0.04$ (Rea et al. 2012). No additional objects were found to be consistent with the Swift-XRT position up to a magnitude $m_{z}=22.2 \pm 0.2(3 \sigma)$.

In addition, data from the UK Infrared Deep Sky Survey (UKIDSS) for the field of SGR 1822-1606 were found to be available, giving the magnitudes of the three aforementioned sources in the $J, H$, and $K$ bands; $m_{J, i}=(13.92,16.62,16.43)$, $m_{H, i}=(12.37,15.75,15.40)$, and $m_{K, i}=(11.62,15.20,14.88)$, where the index $i$ indicates the values for the sources $S 1, S 2$, and $S 3$. In addition to $S 1, S 2$, and $S 3$, no sources were detected within the consistent position up to the limiting magnitudes $m_{J}=19.3, m_{H}=18.3$, and $m_{K}=17.3(5 \sigma)$.

We repeat the same analysis for SGR $0418+5729$ for the case of SGR 1822-1606. We consider only the upper limits since the three sources reported in Rea et al. (2012), S1, S2, and S3, are too luminous to be a white dwarf at the distance considered for the source, $d \approx 5 \mathrm{kpc}$. From the column density value, $N_{H}=7 \times$ $10^{21} \mathrm{~cm}^{-2}$, we obtain an extinction in the $V$-band of $A_{V}=3.89$. From the Cardelli et al. (1989) relation, we obtain the extinction values for the four bands considered: $A_{z}=1.86, A_{J}=1.10$, $A_{H}=0.74$, and $A_{K}=0.44$. The extinction-corrected upper limits do not put very strong constraints on the temperature and the radius of the white dwarf due to the very large distance assumed for SGR 1822-1606. We show in Fig 4 the expected extinctioncorrected magnitudes for a white dwarf with a temperature $T=$ $10^{4} \mathrm{~K}$ and a radius $R=1.5 \times 10^{8} \mathrm{~cm}$ at a distance of $5 \mathrm{kpc}$. We obtain a very deep value for the $K$-band of $\approx 30$. We conclude that, if SGR $1822-1606$ is at the distance of $5 \mathrm{kpc}$ assumed by Rea et al. (2012), it will be hard to detect the white dwarf. On the contrary, a possible detection would lead to a more precise determination of the distance.

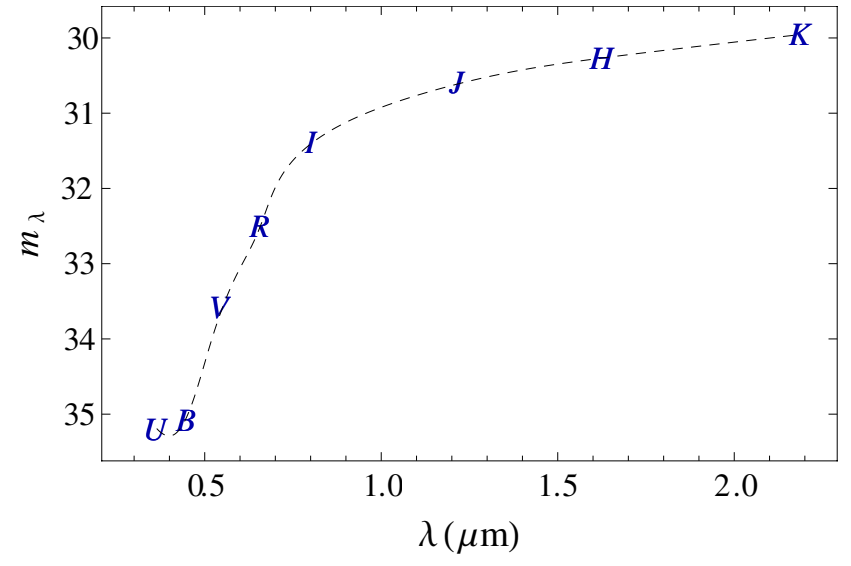

Fig. 4. Expected optical magnitudes of SGR 1822-1606, assuming a blackbody spectral emission from a white dwarf with surface temperature $T=10^{4} \mathrm{~K}$ and a radius of $1.5 \times 10^{8} \mathrm{~cm}$.

\section{1E 2259+586}

\subsection{Bounds of the white dwarf parameters}

In addition to being considered as the AXP prototype, 1E $2259+586$ is the source on which Morini et al. (1988) and Paczynski (1990) based their pioneering idea of describing AXPs as massive, fast-rotating, and highly magnetized white dwarfs. This source is pulsating in X-rays with a period of $P=6.98$ s (Fahlman \& Gregory 1981), has a spindown rate of $\dot{P}=4.8 \times 10^{-13}$ (Davies et al. 1990), and emits X-rays with a luminosity of $L_{\mathrm{X}}=1.8 \times 10^{34} \mathrm{erg} \mathrm{s}^{-1}$ (Gregory \& Fahlman 1980; Hughes et al. 1981; Morini et al. 1988). The corresponding rotation angular velocity of the source is $\Omega=2 \pi / P=0.90 \mathrm{rad} \mathrm{s}^{-1}$. The obtained bounds for the mass, equatorial radius, and moment of inertia of 1E $2259+586$ are shown in Table 3.

\subsection{Solidification and glitches}

The mean density of SGR $1822-1606$ is in the range $3.9 \times 10^{6} \lesssim$ $\bar{\rho} \lesssim 5.9 \times 10^{8} \mathrm{~g} \mathrm{~cm}^{-3}$. The crystallization temperature for such a range following Eq. (4) is then in the range $5.7 \times 10^{6} \mathrm{~K} \lesssim T_{\text {cry }} \lesssim$ $3.0 \times 10^{7} \mathrm{~K}$, which indicates that SGR $1822-1606$ will likely behave as a rigid solid body.

For the minimum and maximum mass configurations and the spindown rate $\dot{P}=4.8 \times 10^{-13}$, we obtain a lower limit for recurrence time of starquakes of

$\delta t_{\mathrm{q}}>\left\{\begin{array}{lll}4.5 \times 10^{7}(|\Delta P| / P) & \mathrm{yr}, & M=M_{\min } \\ 1.2 \times 10^{10}(|\Delta P| / P) & \mathrm{yr}, & M=M_{\max },\end{array}\right.$

which for a typical fractional change of period $|\Delta P| / P \sim 10^{-6}$ gives $\delta t_{\mathrm{q}}>45 \mathrm{yr}$ and $\delta t_{\mathrm{q}}>1.2 \times 10^{4} \mathrm{yr}$, for $M_{\min }$ and $M_{\max }$, respectively. This recurrence time for starquakes is much shorter 
Table 3. Bounds on the properties of $1 \mathrm{E} 2259+586$.

\begin{tabular}{lcccccccccc}
\hline \hline Composition & $M_{\min }$ & $M_{\max }$ & $R_{\mathrm{eq}}^{\min }$ & $R_{\mathrm{eq}}^{\max }$ & $\bar{R}_{\min }$ & $\bar{R}_{\max }$ & $I_{48}^{\min }$ & $I_{50}^{\max }$ & $B_{\min }$ & $B_{\max }$ \\
\hline Helium & 1.28 & 1.41 & 1.15 & 5.94 & 1.15 & 5.39 & 3.56 & 1.12 & 1.26 & 2.30 \\
Carbon & 1.24 & 1.39 & 1.04 & 5.88 & 1.04 & 5.34 & 2.84 & 1.08 & 1.27 & 2.76 \\
Oxygen & 1.23 & 1.38 & 1.08 & 5.86 & 1.08 & 5.32 & 3.05 & 1.52 & 1.52 & 2.60 \\
Iron & 1.00 & 1.11 & 2.23 & 5.49 & 2.21 & 4.98 & 13.1 & 0.78 & 1.33 & 0.62 \\
\hline
\end{tabular}

Notes. The masses $M_{\min , \max }$ are in $M_{\odot}$, the equatorial $R_{\mathrm{eq}}^{\min , \max }$ and mean $\bar{R}_{\min , \max }$ radii area in units of $10^{8} \mathrm{~cm}$, the moments of inertia $I_{48}^{\min }$ and $I_{50}^{\max }$ are in units of $10^{48} \mathrm{~g} \mathrm{~cm}^{2}$ and $10^{50} \mathrm{~g} \mathrm{~cm}^{2}$, respectively. The magnetic fields $B_{\min }$ and $B_{\max }$ are in units of $10^{8} \mathrm{G}$ and $10^{9} \mathrm{G}$, respectively.

than the ones of SGR $0418+5729$ and SGR 1822-1606, indicating that $1 \mathrm{E} 2259+586$ is a very active source in which glitches and outburst activity, such as those observed in 2002 (Kaspi et al. 2003; Woods et al. 2004), can occur with relatively high frequency. It is interesting to note that glitches of minor intensity $|\Delta P| / P \lesssim 10^{-7}$ can be even more frequent, with recurrence times of $\lesssim 4$ yr.

\subsection{Rotation power and magnetic field}

Using the observed values of $P$ and $\dot{P}$, we obtain from Eq. (2) a rotational energy loss

$\left|\dot{E}_{\text {rot }}\right| \approx\left\{\begin{array}{lll}1.6 \times 10^{35} & \operatorname{erg~s}^{-1}, & M=M_{\max } \\ 6.0 \times 10^{36} & \operatorname{erg~s}^{-1}, & M=M_{\min },\end{array}\right.$

which is much larger than the observed X-ray luminosity, $L_{\mathrm{X}}=$ $1.8 \times 10^{34} \mathrm{erg} \mathrm{s}^{-1}$, assuming a distance of $3.2 \pm 0.2 \mathrm{kpc}$ (Kothes \& Foster 2012).

The surface magnetic field of 1E 2259+586 inferred from Eq. (3) is (see Table 3)

$B=\left\{\begin{array}{lll}1.3 \times 10^{8} & \mathrm{G}, & M=M_{\min } \\ 2.8 \times 10^{9} & \mathrm{G}, & M=M_{\max }\end{array}\right.$

Corresponding to the above magnetic fields, the electron cyclotron frequencies are

$v_{\text {cyc }, \mathrm{e}}=\left\{\begin{array}{lll}3.6 \times 10^{14} & \mathrm{~Hz}, & M=M_{\min } \\ 7.8 \times 10^{15} & \mathrm{~Hz}, & M=M_{\max }\end{array}\right.$

which correspond to wavelengths 0.8 and $0.04 \mu \mathrm{m}$, respectively.

\subsection{Optical spectrum and luminosity}

Using data from the Keck telescope, Hulleman et al. (2001) established a faint near-IR counterpart of $1 \mathrm{E} 2259+586$ with a magnitude $K_{\mathrm{s}}=21.7 \pm 0.2$, which is consistent with the position given by Chandra. In addition, upper limits were placed in the optical bands $R=26.4, I=25.6$, and $J=23.8$. From the column density obtained by Patel et al. (2001), $N_{H}=$ $9.3 \pm 0.3 \times 10^{21} \mathrm{~cm}^{-2}$, and using again the empirical formula described by Predehl \& Schmitt (1995), we obtain the absorption $A_{V}=N_{H} /\left(1.79 \times 10^{21} \mathrm{~cm}^{-2}\right)$, which Hulleman et al. (2001) used to obtain the extinction in the other bands $A_{R}=4.3, A_{I}=3.1$, $A_{J}=1.4, A_{K}=0.6$.

It is known that the emission in the $K$ band, the excess in the near-IR, is typically produced by the presence of a disk; see for instance, Hulleman et al. (2000) for the case of 4U 0142+61 and Hulleman et al. (2001) for the present source 1E2259+586, although both are in the context of a fallback disk around a neutron star. We fit the spectrum of $1 \mathrm{E} 2259+586$ as the sum of a blackbody component

$F_{\mathrm{BB}}=\pi \frac{2 h}{c^{2}}\left(\frac{R_{\mathrm{WD}}}{d}\right)^{2} \frac{v^{3}}{e^{h v /\left(k_{B} T\right)}-1}$,

where $R_{\mathrm{WD}}$ and $T$ are the radius and effective temperature of the white dwarf, and a passive, flat, opaque dust disk (see e.g., Jura 2003; Lorén-Aguilar et al. 2009)

$F_{\text {disk }}=12 \pi^{1 / 3} \cos i\left(\frac{R_{\mathrm{WD}}}{\mathrm{d}}\right)^{2}\left(\frac{2 k_{B} T}{3 h v}\right)^{8 / 3}\left(\frac{h v^{3}}{c^{2}}\right) \int_{x_{\text {in }}}^{x_{\text {out }}} \frac{x^{5 / 3}}{e^{x}-1} \mathrm{~d} x,(28)$

where $i$ is the inclination angle of the disk, which we assume as zero degrees, $x_{\text {in }}=h v /\left(k_{B} T_{\text {in }}\right), x_{\text {in }}=h v /\left(k_{B} T_{\text {out }}\right)$, with $T_{\text {in }}$ and $T_{\text {out }}$ the temperatures of the disk at the inner and outer radii, respectively. These temperatures are related to the radii $R_{\text {in }}$ and $R_{\text {out }}$ of the disk through $T_{\text {in,out }}=(2 / 3 \pi)^{1 / 4}\left(R_{\mathrm{WD}} / R_{\text {in,out }}\right)^{3 / 4} T$.

The total flux is then given by $F_{\mathrm{BB}+\text { disk }}=F_{\mathrm{BB}}+F_{\text {disk }}$. Since we have only one point from the observational data, the flux in the $K_{\mathrm{S}}$ band, it is difficult to place constraints on the spectrum parameters. However, we can use the fact that the emission has to respect the upper limits in the $R, I$, and $J$ bands. We have fixed the radius of the white dwarf as $R_{\mathrm{WD}}=3 \times 10^{8} \mathrm{~cm}$, a value in the interval of stability of Table 3 , and for the outer radius of the passive disk we give a typical value $R_{\text {out }}=R_{\odot}$. We found that good fitting values of the other parameters are $T=7.0 \times 10^{4} \mathrm{~K}$ and $T_{\text {in }}=2.0 \times 10^{3} \mathrm{~K}$. In Fig. 5 we show the observed spectrum of $1 \mathrm{E} 2259+586$ in the IR, optical, and UV bands and the composite blackbody + disk model spectrum. The knowledge of more data besides the $K_{\mathrm{s}}$ band will lead to a definite determination of the parameter of the model and to confirmation of the white dwarf nature of 1E $2259+586$.

It is worth recalling that the location of $1 \mathrm{E} 2259+586$ appears to be associated with the supernova remnant G109.1-1.0 (CTB 109) whose age is estimated to be $t-t_{0}=(12-17) \mathrm{kyr}$ (Gregory \& Fahlman 1980; Hughes et al. 1981). Paczynski (1990) proposed a merger of a binary of $\sim(0.7-1) M_{\odot}$ white dwarfs, which leads both to the formation of a fast-rotating white dwarf and to the supernova remnant. Recent simulations of $\left(0.8-0.9 M_{\odot}\right)$ white dwarf mergers (see, e.g., Pakmor et al. 2010) point however to supernova events below the observed explosion energy $\sim 7.4 \times 10^{50} \mathrm{erg}$ of G109.1-1.0 (see, e.g., Sasaki et al. 2004). These events are not very efficient energetically. Additionally, Malheiro et al. (2012) advanced the interesting possibility that this system could be originated from a tight binary system composed of a white dwarf and a late-evolved companion star, thus approaching the process of gravitational collapse. The collapse of the companion star, either to a neutron star or to a black hole, leads, to mass loss which can unbind the original binary system. If the loss of mass in the supernova explosion is $M_{\text {loss }}<M / 2$ with $M$ the total mass of the binary (see, e.g., Ruffini 1973), the system holds bound and the object will therefore lie close to the center of the supernova remnant. 


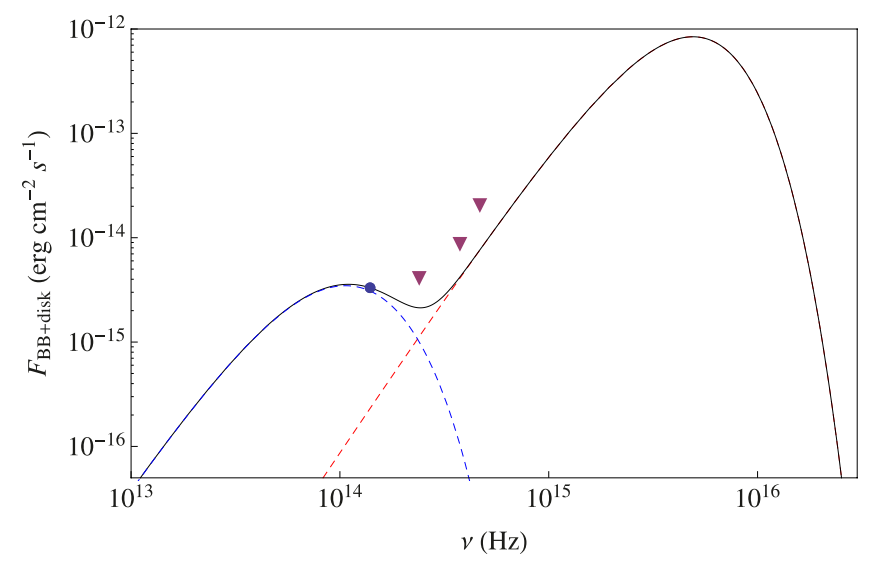

Fig. 5. Observed and fitted spectrum of 1E $2259+586$. The filled circle is the observed flux in the $K_{\mathrm{s}}$ band, and the triangles are the upper limits in the $R, I$, and $J$ bands. The parameters of the blackbody+disk spectrum are $R_{\mathrm{WD}}=3.0 \times 10^{8} \mathrm{~cm}, T=7.0 \times 10^{4} \mathrm{~K}, T_{\text {in }}=2.0 \times 10^{3} \mathrm{~K}$, and $R_{\text {out }}=R_{\odot}$. The blue-dashed curve is the contribution of the disk and the red-dashed curve is the contribution from a pure blackbody. The total spectrum is represented by the solid black curve.

Both explanations are interesting and deserve further investigation. These two scenarios may well explain the presence of a disk of material around the white dwarf, either as material expelled from the white dwarf binary merger (see, e.g., Lorén-Aguilar et al. 2009) in the scenario of Paczynski (1990) or as material coming from the supernova explosion, which is captured by the white dwarf in the scenario of Malheiro et al. (2012). As we have seen, the presence of such a disk explains the emission in the near-IR observed in sources such as 4 U $0142+61$ (see, e.g., Hulleman et al. 2000) and in the present case of 1E 2259+586.

\section{Concluding remarks}

We have described SGR 0418+5729, Swift J1822.3-1606, and $1 \mathrm{E} 2259+586$ as massive, fast-rotating, highly magnetized white dwarfs. The reasons for the choice of these three sources are twofold: 1) the observations of SGR $0418+5729$ (Rea et al. 2010), $P=9.08$ and $\dot{P}<6.0 \times 10^{-15}$, and more recently the ones of Swift J1822.3-1606 (Rea et al. 2012), $P=8.44 \mathrm{~s}$ and $\dot{P}=9.1 \times 10^{-14}$, challenge the description of these sources as ultramagnetized neutron stars, as required by the magnetar model; 2) 1E $2259+586$ is considered the AXP prototype with very good observational data, including the best example of the glitch-outburst connection (see, e.g., Woods et al. 2004). In addition, it represents a historical object, being the one analyzed by Morini et al. (1988) and Paczynski (1990), where the canonical description based on white dwarfs was proposed.

We have shown that SGR $0418+5729$ and Swift J1822.31606 are in full agreement with massive, fast-rotating, highly magnetized white dwarfs. We have improved the white dwarf parameters given by Malheiro et al. (2012) for these sources and also for $1 \mathrm{E} 2259+586$. From an analysis of the rotational stability of Boshkayev et al. (2013), we have given bounds for the mass, radius, moment of inertia, and magnetic field of these sources; see Tables 1-3 for details.

We have improved the theoretical prediction of the lower limit for the spindown rate of SGR $0418+5729$, for which only the upper limit, $\dot{P}<6.0 \times 10^{-15}$, is currently known (Rea et al. 2010). Based on a white dwarf of fiducial parameters, Malheiro et al. (2012) predicted for SGR $0418+5729$ the lower limit $\dot{P}>$ $1.18 \times 10^{-16}$. Our present analysis, which is based on realistic, general relativistic, rotating white dwarfs allows us to improve this prediction; see Eq. (10) in Sect. 4.4 for the new numerical values. In the case of a white dwarf close to the critical mass, this new lower limit gives a very stringent constraint on the spindown rate of SGR $0418+5729, \dot{P}=4 \times 10^{-16}<\dot{P}<6 \times 10^{-15}$, which we submit for observational verification. Indeed, after the submission of this work, Rea et al. (2013) reported the confirmation of the spindown rate of SGR $0418+5729, \dot{P}=4 \times 10^{-15}$, at 3.5 sigma confidence level. This measurement fully confirms the results of Malheiro et al. (2012), see Eq. (1), as well as the more stringent constraints presented in this work, see Eq. (10). Since these constraints are presented in advance to the observations, they have to be considered as predictions. This fact clearly represents an observational support for the white dwarf model of SGRs/AXPs.

Along these lines it is worth mentioning the recent discussions on the high uncertainties and different results claimed by different authors on the value of the first period time derivative of Swift J1822.3-1606 (see Tong \& Xu 2012 and references therein for details). Here we have used the value reported by Rea et al. (2012). However, it would also be interesting in this case to put a theoretical lower limit with the white dwarf mode. Using $L_{\mathrm{X}}=4 \times 10^{32} \mathrm{erg} \mathrm{s}^{-1}$ at a distance of $5 \mathrm{kpc}$ (Rea et al. 2012), we obtain a lower limit $\dot{P} \geq L_{X} P^{3} /\left(4 \pi^{2} I\right) \approx 2.13 \times 10^{-15}$ for a ${ }^{12} \mathrm{C}$ white dwarf close to its maximum mass; see Table 3 . Indeed, this limit bounds from below all the observationally claimed spindown rates for this source known up to now.

We gave in Eqs. (9), (22), and (26) an additional prediction of the frequencies at which absorption features could be present in the spectra of SGR 0418+5729, Swift J1822.3-1606, and $1 \mathrm{E} 2259+586$, respectively, as a result of the scattering of photons with electrons whose energy spectrum is quantized due to the magnetic field. The range we obtained for such frequencies falls between the infrared and UV bands. It is important to remark that magnetic fields in white dwarfs ranging from $10^{7} \mathrm{G}$ up to $10^{9} \mathrm{G}$ are routinely observed; see, e.g., Külebi et al. (2009), Külebi et al. (2010a), Kepler et al. (2010), and very recently Kepler et al. (2013), where from the Data Release 7 of the Sloan Digital Sky Survey, white dwarfs with magnetic fields in the range from around $10^{6} \mathrm{G}$ to $7.3 \times 10^{8} \mathrm{G}$ have been found from the analysis of the Zeeman splitting of the Balmer absorption lines. Deep photometric and spectrometric observations in the range of cyclotron frequencies predicted in this work are therefore highly recommended to detect possible absorptions and line-splitting features in the spectra of SGRs and AXPs.

We have presented the optical properties of SGR 0418+ 5729, Swift J1822.3-1606, and 1E 2259+586, as expected from a model based on white dwarfs. We inferred the surface temperature and predicted the emission fluxes in the UV, optical, and IR bands. We have shown that indeed the available observational data are consistent with a white dwarf model for these objects. In the particular case of $1 \mathrm{E} 2259+586$, the observed excess in the near-IR is explained by the presence of a disk of dust around the white dwarf. Such a disk might be the result of material expelled during the merger of a white dwarf binary progenitor (Paczynski 1990; Rueda et al. 2013) or as the result of material from the supernova explosion of an evolved star companion of the white dwarf in the binary scenario proposed by Malheiro et al. (2012) for the SGR/AXP-supernova connection.

It is important to discuss briefly the persistent X-ray emission of SGRs/AXPs. The time integrated X-ray spectrum is often well described by a composite blackbody + power-law model with temperatures of the order of $k_{\mathrm{B}} T_{\mathrm{BB}} \sim 0.1 \mathrm{keV}$ (see, e.g., Göhler et al. 2005). Such a blackbody component corresponds 
to temperatures $T_{\mathrm{BB}} \sim 10^{6} \mathrm{~K}$, which are higher than the surface temperature of a white dwarf (i.e., such as the ones predicted in this work). This clearly points to an X-ray emission of magnetospheric origin, and so this blackbody temperature of the X-ray spectrum is not to be associated with the white dwarf effective temperature (see, e.g., Malheiro et al. 2012). A possible mechanism for the X-ray quiescent emission from a magnetized white dwarf was underlined by Usov (1993): the reheating of the magnetosphere owing to the bombardment of the backward moving positrons, which were created in the pair cascades formed in the interaction of the high-energy photons with the ultra-relativistic electrons. As shown by Usov (1993) in the specific case of $1 \mathrm{E} 2259+586$, such a reheating of the polar caps is able to produce a stable $X$-ray luminosity $L_{X} \sim 10^{35} \mathrm{erg} \mathrm{s}^{-1}$, in agreement with observations.

A well-known observational problem of SGRs and AXPs is the uncertainty in the estimation of the distances of the sources (see, e.g., Kothes \& Foster (2012) for a critical discussion on the distance of 1E2259+586). These uncertainties strongly affect the estimates of the interstellar reddening $A_{V}$, which are crucial for the precise calculation of the source properties and therefore for a clear identification of the nature of the compact object. Deeper observations of Hubble and VLT are thus strongly recommended to establish the precise values of the luminosity in the optical and in the near-IR bands, which will verify the white dwarf nature of SGRs and AXPs.

We encourage future observational campaigns from space and ground to verify all the predictions presented in this work.

Acknowledgements. We thank the referee, Alexander Potekhin, for the comments and suggestions, which helped us to improve the presentation of our results.

\section{References}

Barstow, M. A., Jordan, S., O’Donoghue, D., et al. 1995, MNRAS, 277, 971 Baym, G., \& Pines, D. 1971, Ann. Phys., 66, 816

Boshkayev, K., Rueda, J. A., Ruffini, R., \& Siutsou, I. 2013, ApJ, 762, 117

Cardelli, J. A., Clayton, G. C., \& Mathis, J. S. 1989, ApJ, 345, 245

Davies, S. R., Coe, M. J., \& Wood, K. S. 1990, MNRAS, 245, 268

Duncan, R. C., \& Thompson, C. 1992, ApJ, 392, L9

Durant, M., Kargaltsev, O., \& Pavlov, G. G. 2011, ApJ, 742, 77

Fahlman, G. G., \& Gregory, P. C. 1981, Nature, 293, 202

Ferrari, A., \& Ruffini, R. 1969, ApJ, 158, L71

Ferrario, L., Wickramasinghe, D., Liebert, J., \& Williams, K. A. 2005, MNRAS, 361,1131
Friedman, J. L., Ipser, J. R., \& Sorkin, R. D. 1988, ApJ, 325, 722

Göhler, E., Wilms, J., \& Staubert, R. 2005, A\&A, 433, 1079

Gregory, P. C., \& Fahlman, G. G. 1980, Nature, 287, 805

Hartle, J. B. 1967, ApJ, 150, 1005

Hartle, J. B., \& Thorne, K. S. 1968, ApJ, 153, 807

Hughes, V. A., Harten, R. H., \& van den Bergh, S. 1981, ApJ, 246, L127

Hulleman, F., van Kerkwijk, M. H., \& Kulkarni, S. R. 2000, Nature, 408, 689

Hulleman, F., Tennant, A. F., van Kerkwijk, M. H., et al. 2001, ApJ, 563, L49

Jura, M. 2003, ApJ, 584, L91

Kaspi, V. M., Gavriil, F. P., Woods, P. M., et al. 2003, ApJ, 588, L93

Kepler, S. O., Kleinman, S. J., Pelisoli, I., et al. 2010, in AIP Conf. Ser. 1273, eds. K. Werner, \& T. Rauch, 19

Kepler, S. O., Pelisoli, I., Jordan, S., et al. 2013, MNRAS, 429, 2934

Kothes, R., \& Foster, T. 2012, ApJ, 746, L4

Külebi, B., Jordan, S., Euchner, F., Gänsicke, B. T., \& Hirsch, H. 2009, A\&A, 506, 1341

Külebi, B., Jordan, S., Euchner, F., Gaensicke, B. T., \& Hirsch, H. 2010a, VizieR Online Data Catalog: JIA+A/1506/1341

Külebi, B., Jordan, S., Nelan, E., Bastian, U., \& Altmann, M. 2010b, A\&A, 524, A36

Liebert, J., Schmidt, G. D., Green, R. F., Stockman, H. S., \& McGraw, J. T. 1983, ApJ, 264, 262

Lorén-Aguilar, P., Isern, J., \& García-Berro, E. 2009, A\&A, 500, 1193

Malheiro, M., Rueda, J. A., \& Ruffini, R. 2012, PASJ, 64, 56

Malov, I. F. 2010, Astron. Rep., 54, 925

Mereghetti, S. 2008, A\&AR, 15, 225

Morini, M., Robba, N. R., Smith, A., \& van der Klis, M. 1988, ApJ, 333, 777

Paczynski, B. 1990, ApJ, 365, L9

Pagani, C., Beardmore, A. P., \& Kennea, J. A. 2011, ATel., 3493, 1

Pakmor, R., Kromer, M., Röpke, F. K., et al. 2010, Nature, 463, 61

Patel, S. K., Kouveliotou, C., Woods, P. M., et al. 2001, ApJ, 563, L45

Predehl, P., \& Schmitt, J. H. M. M. 1995, A\&A, 293, 889

Qadir, A., Ruffini, R., \& Violini, G. 1980, Nuovo Cimento Lettere, 27, 381

Rea, N., Esposito, P., Turolla, R., et al. 2010, Science, 330, 944

Rea, N., Israel, G. L., Esposito, P., et al. 2012, ApJ, 754, 27

Rea, N., Israel, G. L., Pons, J. A., et al. 2013, ApJ, 770, 65

Rotondo, M., Rueda, J. A., Ruffini, R., \& Xue, S.-S. 2011, Phys. Rev. C, 83, 045805

Rueda, J. A., Aznar-Siguán, G., Boshkayev, K., et al. 2013, ApJ, submitted

Ruffini, R. 1973, in Black Holes (Les Astres Occlus), ed. A. Giannaras, 451

Salpeter, E. E. 1961, ApJ, 134, 669

Sasaki, M., Plucinsky, P. P., Gaetz, T. J., et al. 2004, ApJ, 617, 322

Schmidt, G. D., West, S. C., Liebert, J., Green, R. F., \& Stockman, H. S. 1986, ApJ, 309, 218

Schmidt, G. D., Bergeron, P., Liebert, J., \& Saffer, R. A. 1992, ApJ, 394, 603

Shapiro, S. L., \& Teukolsky, S. A. 1983, Black holes, white dwarfs, and neutron stars: The physics of compact objects, eds. S. L. Shapiro, \& S. A. Teukolsky

Thompson, C., \& Duncan, R. C. 1995, MNRAS, 275, 255

Tong, H., \& Xu, R. X. 2012 [arXiv: 1212 .4314]

Trümper, J. E., Dennerl, K., Kylafis, N. D., Ertan, Ü., \& Zezas, A. 2013, ApJ, 764,49

Usov, V. V. 1993, ApJ, 410, 761

Usov, V. V. 1994, ApJ, 427, 984

Woods, P. M., Kaspi, V. M., Thompson, C., et al. 2004, ApJ, 605, 378 